\title{
Measurement of T2K Anti-neutrino Beam Properties Using the INGRID On-axis Near Detector
}

\section{T.Hayashino* for T2K collaboration}

Kyoto University

E-mail: t.hayashino@scphys.kyoto-u.ac.jp

\begin{abstract}
In the $\mathrm{T} 2 \mathrm{~K}$ experiment, neutrino oscillations are measured by using the J-PARC neutrino beam, the near detectors at J-PARC and the far detector, Super-Kamiokande, at Kamioka. The T2K experiment uses a highly pure muon neutrino beam and measures neutrino oscillation parameters. $\mathrm{T} 2 \mathrm{~K}$ made the first observation of electron neutrino appearance in a muon neutrino beam and constrained CP-violating phase $\delta_{C P}$. Recently T2K started taking data using anti-neutrino beam to enhance the sensitivity to measure $\mathrm{CP}$ violation in 2014. This is the first anti-neutrino beam operation for $\mathrm{T} 2 \mathrm{~K}$ and it will be very important to measure the beam properties using the near detectors. The INGRID on-axis near detector measures the neutrino beam profile center and intensity using 14 identical modules. In this paper we report the anti-neutrino beam measurements using the INGRID anti-neutrino run data taken up to March 2015.
\end{abstract}

Flavor Physics $\mathcal{E}$ CP Violation 2015,

May 25-29, 2015

Nagoya, Japan

${ }^{*}$ Speaker. 


\section{Introduction}

In the T2K experiment, neutrino oscillations are measured using the highly pure muon neutrino beam from J-PARC, the near detectors at J-PARC and the far detector, Super-Kamiokande, at Kamioka.

T2K is the world's first long baseline neutrino experiment which adapted the off-axis beam method. The off-axis near detector and Super Kamiokande are positioned 2.5 degrees away from the center direction of the neutrino beam (Fig W). The off-axis beam has a narrow-band energy spectrum and the off-axis angle is optimized to maximize the neutrino oscillation probability at Super-Kamiokande. The neutrino energy spectrum is sensitive to the beam direction, so it is important to monitor and control the beam direction precisely.

The $30 \mathrm{GeV}$ proton beam is generated at J-PARC main ring and strikes a graphite target and produces secondary particles. Then, the charged secondary particles are focused by three magnetic horns. When the $\pi^{+}\left(\pi^{-}\right)$particles are focused, a muon (anti-muon) neutrino beam is produced from the decay products. T2K started taking data using anti-neutrino beam to enhance the sensitivity of its CP violation measurement in June 2014. This is the first anti-neutrino beam operation for T2K.

\section{INGRID}

INGRID is an on-axis neutrino near detector located at $280 \mathrm{~m}$ downstream from the graphite target (Fig W). The main purpose of INGRID is to monitor the neutrino beam profile center with a precision better than $28 \mathrm{~cm}$ which corresponds to $1 \mathrm{mrad}$ of the beam direction at INGRID location $(280 \mathrm{~m} \times 1 \mathrm{mrad})[\square]$. INGRID consists of $14+2$ identical modules (Fig \). We only use 14 modules arranged in horizontal and vertical arrays around the beam center to monitor the beam properties. Each module consists of nine iron target planes and eleven tracking scintillator planes which are surrounded by VETO scintillator planes to reject charged particles coming from outside the modules. The dimensions of the iron target plates are $124 \times 124 \mathrm{~cm}^{2}$ in the horizontal and vertical directions and $6.5 \mathrm{~cm}$ along the beam direction.

Each scintillator bar has a hole of about $3 \mathrm{~mm}$ in diameter at the center and a wavelength shifting fiber is inserted in the hole for the light collection. The fiber is attached to the Multi Pixel Photon Counter (MPPC).

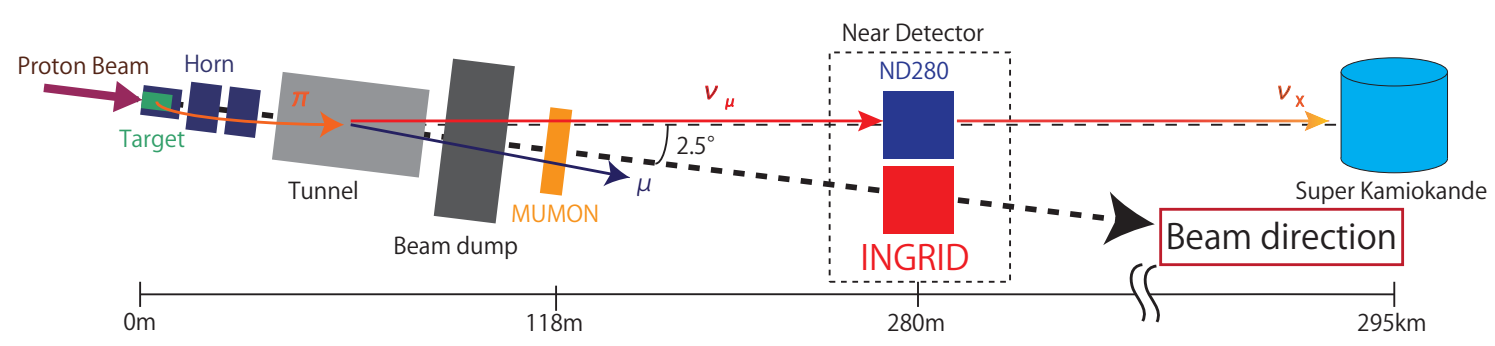

Figure 1: Schematic view of the T2K beam line. 


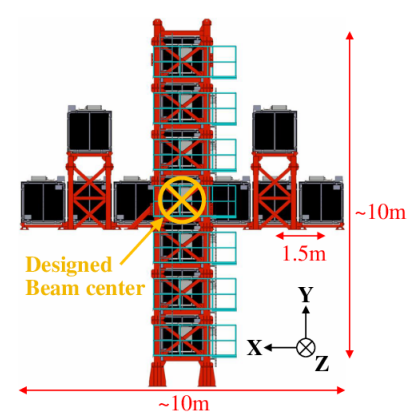

Figure 2: Schematic drawig the T2K INGRID detector

\section{Event Selection}

INGRID reconstructs tracks of secondary charged particles, mainly $\mu$, generated by neutrino interaction (Fig B]). The event selection of INGRID is described elseware [3]. After this selection, the neutrino detection efficiency is $66 \%$ and the background ratio is $2.67 \%$. Main background sources are neutron and gamma generated in walls of the neutrino detector hall.

\section{Results of the Measurement}

In this proceeding, data used was taken during June 2014 - March 2015. Integrated proton on target (POT) in the anti-neutrino runs is $3.63 \times 10^{20}$. The dead time fraction of INGRID data is lower than $0.01 \%$ in this period. The analyzed data is divided into two periods, one is named T2K Run5 (June 2014), the other is named T2K Run6 (October 2014 - March 2015). The neutrino beam profiles are fit with a Gaussian and its central values are defined as the beam center.

\subsection{Systematic Error}

Three sources of systematic errors on the profile center are considered. They include (1) the detector systematic error, (2) the difference between the actual beam center and the Gaussian fit center, and (3) the uncertainties in the survey of the detector position. In this analysis, the detector systematic error is $4.08 \%$ and other errors are negligible.

We performed toy Monte Carlo studies of a million pseudo-experiments on the beam profiles. The number of events at each module is varied by $4.08 \%$ and the beam center is reconstructed with the same method as the data analysis. The RMS of reconstructed center value is taken as the systematic error.

\subsection{Event Rate and Beam Profile}

The event rate which is the number of neutrino events in 14 INGRID modules is normalized by $10^{14} \mathrm{POT}$. The anti-neutrino beam event rates are $0.562 \pm 0.001$ (stat.) [events $/ 10^{14} \mathrm{POT}$ ] in T2K Run5 and $0.559 \pm 0.0004$ (stat.) [events $/ 10^{14} \mathrm{POT}$ ] in T2K Run6. The upper plot of Fig $⿴ 囗 十$ is a day-by-day event rate plot. Daily event rates are stable at around 0.56 events $/ 10^{14} \mathrm{POT}$.

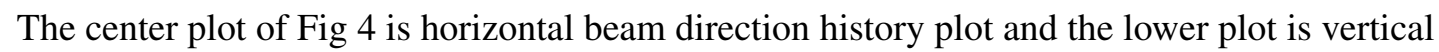
beam position history plot. Table $\square$ shows the event rate and center of the neutrino beam in the 
Table 1: The center and width of the neutrino beam in the anti-neutrino runs.

\begin{tabular}{|l||r|r|}
\hline \multicolumn{1}{|l||}{ Run period } & T2K Run5 & T2K Run6 (until March) \\
\hline \hline Event rate [events $\left./ 10^{14} \mathrm{POT}\right]$ & $0.562 \pm 0.001$ (stat.) & $0.559 \pm 0.0004$ (stat.) \\
\hline Horizontal beam angle $[\mathrm{mrad}]$ & $0.050 \pm 0.067$ (stat.) \pm 0.357 (syst.) & $0.010 \pm 0.028$ (stat.) \pm 0.363 (syst.) \\
\hline Vertical beam angle [mrad] & $0.155 \pm 0.076$ (stat.) \pm 0.427 (syst.) & $0.102 \pm 0.031$ (stat.) \pm 0.423 (syst.) \\
\hline
\end{tabular}

anti-neutrino runs in each run period. The observed horizontal and vertical beam center shifts are smaller than the physics requirement, $28 \mathrm{~cm}$.

\section{Conclusion}

The T2K experiment succeeded in generating anti-neutrino beam and the INGRID detector recorded $3.63 \times 10^{20}$ POT until March 2015. The INGRID event rate was 0.56 Events $/ 10^{14}$ POT and rate was stable for data taking. The shift in beam direction was within $1 \mathrm{mrad}$ and met the T2K physics requirements.

\section{References}

[1] K. Abe et al. (T2KCollaboration): Phys. Rev. Lett. 112. (2014) 061802.

[2] T2K-ND280: Technical Design Report (2006).

[3] T. Kikawa : Ph.D.thesis, Kyoto University, Measurement of Neutrino Interactions and Three Flavor Neutrino Oscillations in the T2K Experiment. (2014)

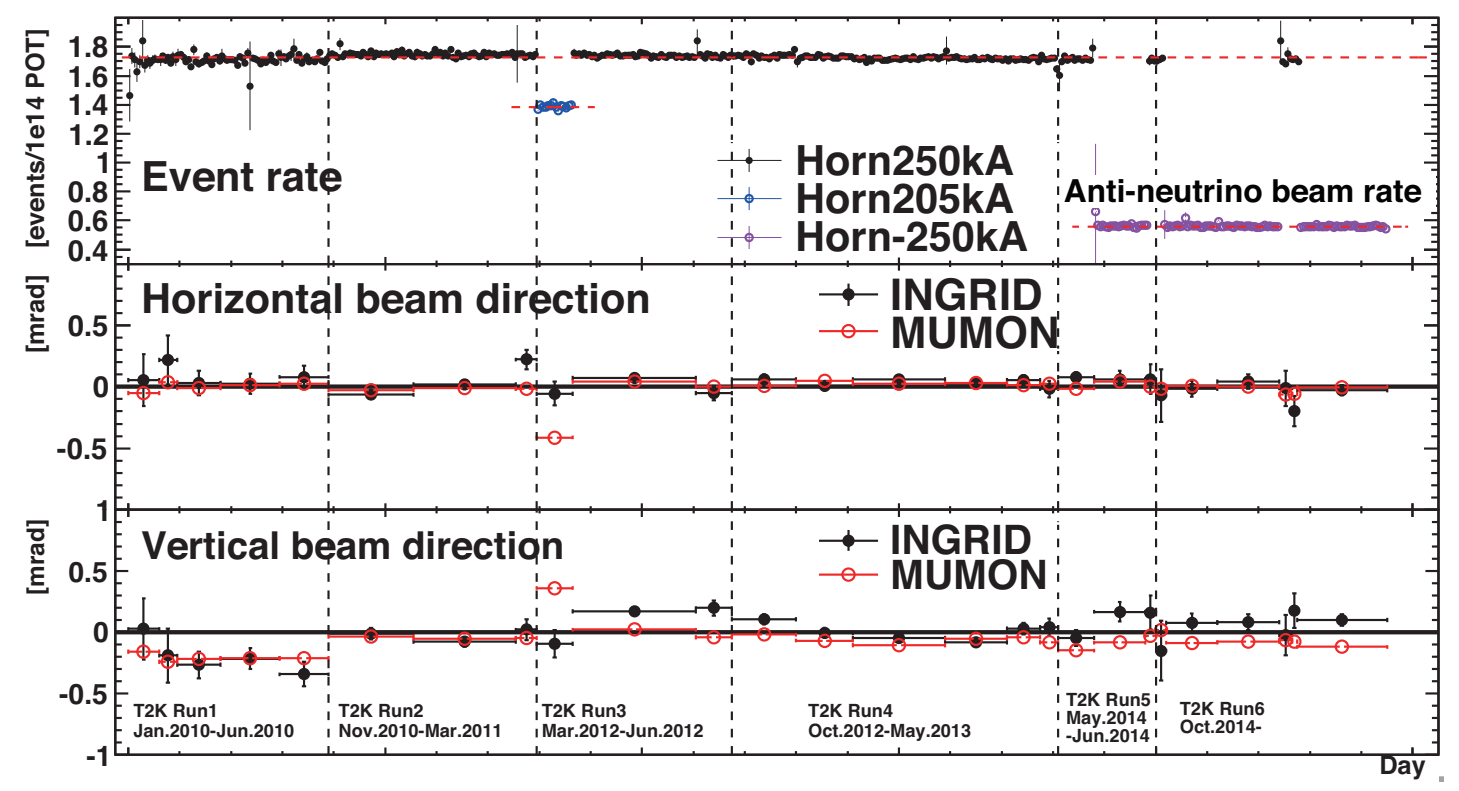

Figure 4: (Upper) event rate in events/10 ${ }^{14}$ POT vs. time, (Middle) horizontal beam direction in mrad and (Lower) vertical beam direction in mrad. 